\title{
Label free technologies: Raman micro- spectroscopy and multi-spectral imaging for lymphocyte classification
}

\author{
Teddy Happillon ${ }^{1 \dagger}$, Valérie Untereiner ${ }^{1 \dagger}$, Abdelilah Beljebbar $^{1 \dagger}$, Cyril Gobinet $^{1 \dagger}$, Michel Manfait ${ }^{1 \dagger}$, Sylvie Daliphard ${ }^{2 \dagger}$, \\ Pascale Cornillet-Lefebvre ${ }^{2 \dagger}$, Xavier Troussard ${ }^{3 \dagger}$, Jesus Angulo ${ }^{4 \dagger}$, Santiago Velasco-Forero ${ }^{4 \dagger}$, Véronique Saada ${ }^{5 \dagger}$, \\ Georges Flandrin ${ }^{6+}$, Jacques Klossa ${ }^{6 *}$
}

From 11th European Congress on Telepathology and 5th International Congress on Virtual Microscopy Venice, Italy. 6-9 June 2012

\section{Background}

Current diagnostic and prognostic approaches in oncology use morphological and molecular techniques which lead to patient personalized therapies. However, they are still complex and hard to standardize. This is also true for Chronic Lymphocytic Leukemia (CLL) that has been chosen for the IHMO project [1,2]. Simplifying diagnostic processes and making easier the standardization would be highly suitable. In order to develop such a simpler automated method, the IHMO project, funded by the French National Research Agency, proposed to develop a multimodal microscopy scanning platform that includes in a single machine a Raman micro-spectrometer (RMS) combined with a multispectral imager (MSI) [3,4].

RMS is a quick non-invasive and non-destructive technique for tissues and cells analysis [5]. It is very sensible to molecular changes and it could be used as a powerful diagnostic and prognostic tool when used in association with multivariate statistical methods. It is particularly useful for characterizing pathological tumors especially at the cellular level $[6,7]$. Multispectral imaging in the visible spectrum could confirm RMS classifications and provide new morphometric findings [8].

CLL disease is characterized by the proliferation of lymphocytes (lymphocytosis). It is the most common leukemia, preferentially affecting people aged over 50 years old. It is incurable and in most cases shows no clinical signs. Thus, it is often discovered by chance during a blood test. If necessary, morphological and immunological studies are

\footnotetext{
* Correspondence: jklossa@tribvn.com

+ Contributed equally

${ }^{6}$ TRIBVN, 39, rue Louveau, 92320 Châtillon, France

Full list of author information is available at the end of the article
}

led by analyzing blood smears colored with May-Grünwald Giemsa, by making a complete blood count and by computing a Matutes score. Such studies are necessary because it is impossible to distinguish a healthy cell from a cancerous one only using a conventional microscope.

\section{Material and methods}

Blood smears were prepared on classical glass slides commonly used in laboratories for microscopy; cells were localized by optical microscopy. A new multimodal machine which has been developed combining i) a 10 bands multispectral imager using the full spectrum of transmitted visible light ii) a Raman micro-spectrometer, equipped with a $532 \mathrm{~nm}$ diode laser excitation source delivering $13.5 \mathrm{~mW}$ of power on the sample; iii) a microscope stand with 40x and 150x lenses suited with an xyz motorized stage for scanning the blood smear, and localizing $x-y$ coordinates of a representative series ( 100 for each patient) of lymphocyte cells before registering Raman spectra on their nuclei and their individual multi-spectral images. An additional piezo actuator allowed for precise $\mathrm{z}$ stack recording. Figure 1 shows each step from the screening of the smears to the final results.

\section{Raman micro-spectroscopy}

More precisely, 997 polymorphonuclears, 95 monocytes and 1127 lymphocytes from 12 different blood samples have been considered in the first part of this study, and a Raman spectrum has been acquired on each of them. In the second part, 4257 spectra have been registered on lymphocytes of 49 leukemic patients suffering from hyper leukocytosis Chronic Lymphocytic Leukemia, and 2596 spectra have been recorded on lymphocytes of 27 healthy 




\section{$A^{\wedge}$ : Hardware and software multimodal platform}

1 - Blood smear region of screening

2 - Registered cells of interest

$3-150 x$ morphology, scale of cell $=8 \mu \mathrm{m}$

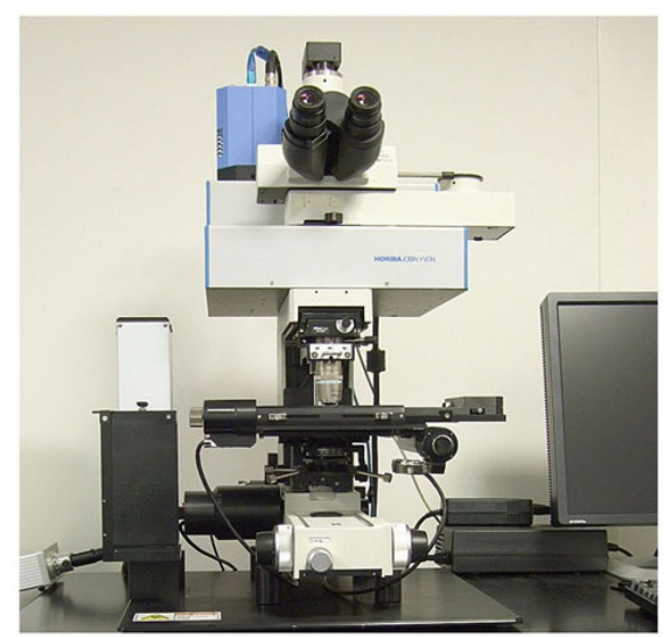

4 - Raman spectrum on nucleus, resolution $x-y=1 \mu \mathrm{m}$

5 - Cells classification / molecular signature
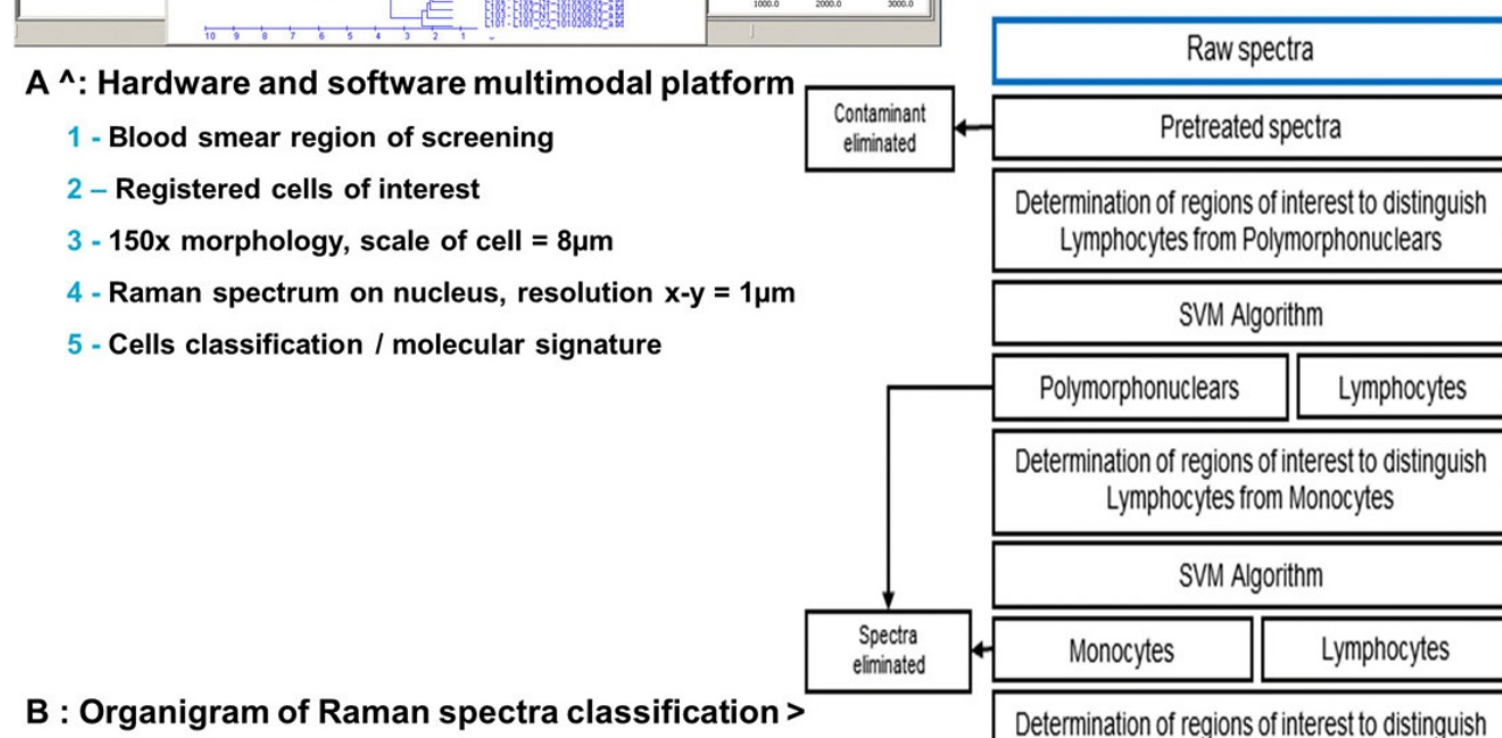

Determination of regions of interest to distinguish Lymphocytes from Polymorphonuclears

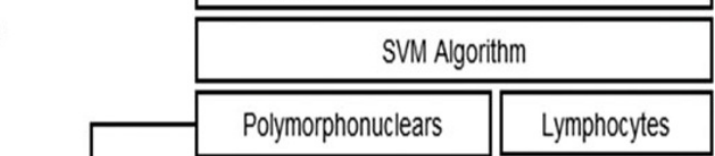

Determination of regions of interest to distinguish Lymphocytes from Monocytes

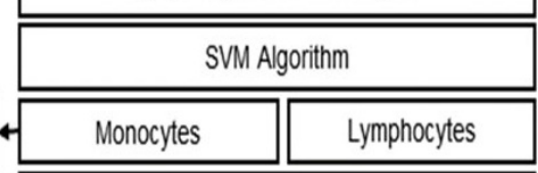

Determination of regions of interest to distinguish Healthy from CLL Iymphocytes

\begin{tabular}{|c|c|}
\hline $\begin{array}{c}\text { Healthy labelled } \\
\text { lymphocytes }\end{array}$ & $\begin{array}{l}\text { CLL labelled } \\
\text { lymphocytes }\end{array}$ \\
\hline
\end{tabular}

Figure $1 \mathrm{IHMO}$ platform includes the Raman micro-spectrometer and the multi-spectral sources (hardware) and a software able to control the acquisitions and the classification process.

individuals. These spectra have been then studied, using around 90 cells per blood sample.

Raman data were first pretreated to erase contaminant information into the spectra and then were analyzed using a multivariate statistical method which put forward the relevant information needed to distinguish in a first time lymphocytes from polymorphonuclears. The spectra are then reduced to their relevant information and classified using a Support Vector Machine algorithm [9]. Then this algorithm was used to develop a classification model splitting leukemic and healthy lymphocytes; 3 sets of data were created, the first one, the training set, composed of the spectra from 6 leukemic patients (513 spectra) and 4 healthy individuals (315 spectra) which was used to create different prediction models, the second set, the validation set, composed of spectra from 33 leukemic patients (2820 spectra) and 13 healthy individuals (1106 spectra) was used to select the best model among all previously computed. Finally, the third set, the test set, composed of spectra from 20 different blood samples (2099 spectra) was used to validate the selected classification model in a blind way. 


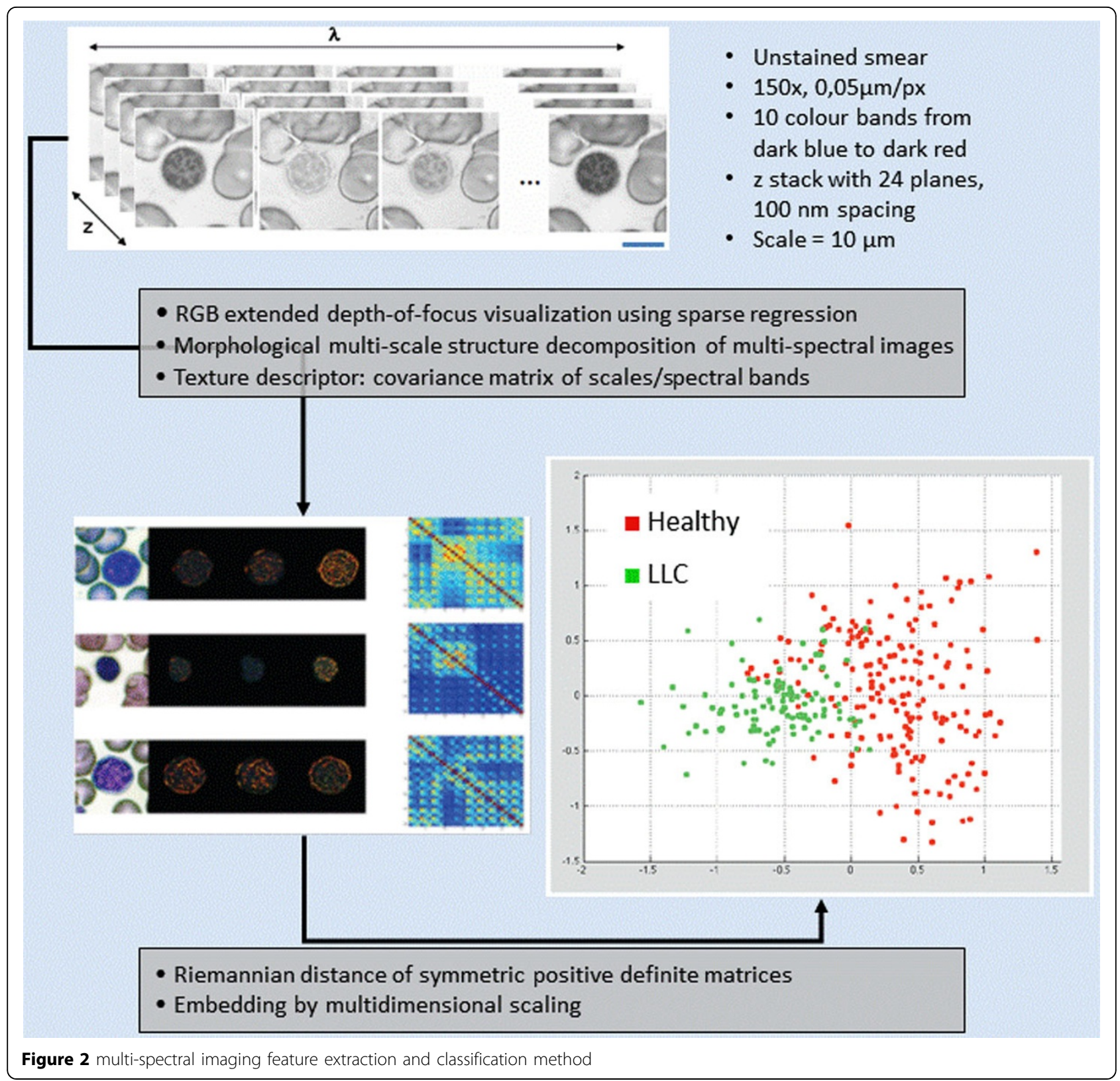

\section{Multi-spectral imaging}

After acquisition of Raman spectra, the slides are stained using a RAL_DIAGNOSTICS ${ }^{\mathrm{TM}}$ standardized staining protocol. Then, for each target cell, a multi-z/multi-spectral image is acquired: a z-stack of $100 \mathrm{~nm}$ spaced of 24 monochrome images for each of the ten wavelength bands. An algorithm combining mathematical morphology techniques and sparse regression was developed to produce a color RGB image with an extended-depth-of-focus from the multi-z/multispectral image. This color image is used to visually confirm the classification of cells characterized by RMS. The set of scalar images multi-Z/multi-spectral of each cell is also processed by mathematical morphology techniques to produce morpho-spectral texture descriptors reducing the 240 images from each color band to a set of 4 representative images. Such data reduction process allowed representing each cell by a $40 \times 40$ square symmetrical correlation matrix. Then, using tools from information geometry and multivariate statistics, cells are embedded into a dimensionality reduced space used to produce an unsupervised classification into two classes: leukemia patients vs. healthy individuals.

\section{Results and discussion}

First stage molecular classification with Raman spectrometry aimed at identifying the nucleated components 
from a blood smear, mainly polymorphonuclears and lymphocytes. Indeed such differentiation is straightforward with morphological methods like MSI even on unstained samples. Molecular classification gave a sensitivity of $99.3 \%$ and a specificity of $99.6 \%$.

The second stage aimed at classifying spectra from leukemic and healthy lymphocytes and provided a sensitivity of $80 \%$ and a specificity of $100 \%$ on an extended set of blind samples.

It has to be mentioned that in a first step of this work, we tried to match the classification results to the percentages obtained with flow cytometry in term of T, B and NK lymphocytes of a blood sample. However, these results could not be compared with each other since in this study the information which is taken into account is obtained from the nucleus of each white blood cell. As a result, this is a molecular and nuclear information while, in classical cytology, this is the membrane of the cells which is considered.

Morpho-spectral texture classification from multispectral imaging in the visible is used to complement and consolidate the RMS data classifications and in on-going work will be used to identify specific morpho-spectral characteristics associated to leukemic cells (see figure 2).

\section{Conclusion}

IHMO project has demonstrated the power of Raman micro-spectroscopy coupled with supervised classification algorithm such as Support Vector Machines for cell classification and more precisely for the diagnosis of CLL. Morphological descriptors obtained from multi- $Z$ and multispectral images provide another independent classification that still needs to be assessed.

The multimodal microscopy platform can be used more generally in the field of cytohematology, however application to cytological and histological pathology would need further developments and could take profit from new methods in data classification.

\section{List of abbreviations}

RMS: Raman Micro-Spectroscopy; MSI: Multi-Spectral Imaging; CLL: Chronic Lymphoid Leukemia; SVM: Support Vector Machines

\section{Competing interests}

The authors declare that they have no competing interests.

\section{Authors' contributions}

- TH carried out the acquisitions, pretreatments, the highlighting of relevant information into the spectra, the classification of the data and drafted the extended abstract.

- $A B$ drove the implementation of pretreatments adapted to spectral data and found a way to highlight the relevant information into the spectra.

- $V U$ realized the acquisitions of the data and drafted the extended abstract. - CG contributes to the choice of the classification algorithm among several studied (supervised or not), and participated in the choice of each possible settings of these algorithms to improve the obtained results.

- JA and SVF developed the multi-spectral classification method and produced the multi-spectral classification.
- XT, JK and MM both designed and managed the full project with the contribution of GF

- XT, SD, VS and PCL selected the best CLL patients, provided the corresponding samples and patient's data, and realized the flow cytometry for each of them.

\section{Acknowledgements}

- The French National Research Agency (ANR) for funding - Chih-Chung Chang and Chih-Jen Lin, LIBSVM: a library for support vector machines, 2001. Software available at http://www.csie.ntu.edu.tw/ cjlin/ libsvm

- Clinical staff of the university hospital of Reims

- Rodolphe Dagiral and Valérie Coomans from Réactifs RAL for developing and providing standardizes MGG stains used for multi-spectral imaging classification

- Philippe Rideau, David Pointu and Claire Vernois from NIKON France for defining and installing appropriate microscopy hardware

- Emmanuel Froigneux from HORIBA JOBIN YVON for developing and providing the micro-spectroscopy Raman module

\section{Authors' details}

${ }^{1}$ MEDyC FRE/CNRS 3481, 51096 Reims, France. ${ }^{2} \mathrm{CHU}$ de Reims, 51100 Reims, France. ${ }^{3} \mathrm{CHU}$ de Caen, 14033 Caen, France. ${ }^{4} \mathrm{CMM}$-ARMINES, 77300

Fontainebleau, France. ${ }^{5}$ IGR-Institut Gustave-Roussy, 94805 Villejuif, France.

${ }^{6}$ TRIBVN, 39, rue Louveau, 92320 Châtillon, France.

Published: 30 September 2013

\section{References}

1. Cramer $\mathrm{P}$, Hallek M: Prognostic factors in chronic lymphocytic leukemia what do we need to know? Nat Rev Clin Oncol 2011, 8(1):38-47.

2. Zenz $T$, Mertens $D$, Küppers $R$, Döhner $H$, Stilgenbauer $S$ : From pathogenesis to treatment of chronic lymphocytic leukaemia. Nat Rev Cancer 2010, 10(1):37-50.

3. Velasco-Forero S, Angulo J: Parameters selection of morphological scale space decomposition for hyperspectral images using tensor modeling. Proc. of SPIE symposium on Defense, Security, and Sensing: Algorithms and Technologies for Multispectral, Hyperspectral, and Ultraspectral Imagery XVI, SPIE 2010, 7695:12.

4. Malik Z, Rothmann C, Cycowitz T, Cycowitz ZJ, Cohen AM: Spectral morphometric characterization of B-CLL cells versus normal small lymphocytes. J Histochem Cytochem 1998, 46(10):1113-8.

5. Anthony T: Raman spectroscopy in biology. NewYork: JohnWiley\&Sons; 1982.

6. Draux F, Jeannesson P, Beljebbar A, Tfayli A, Fourre N, Manfait M, SuléSuso J, Sockalingum GD: Raman spectral imaging of single living cancer cells: a preliminary study. Analyst 2009, 134:542-8.

7. Krishna CM, Sockalingum GD, Vadhiraja BM, Maheedhar K, Rao AC, Rao L, Venteo L, Pluot M, Fernandes DJ, Vidyasagar MS, Kartha VB, Manfait M: Vibrational spectroscopy studies of formalin-fixed cervix tissues. Biopolymers 2007, 85:214-21.

8. Angulo J, Klossa J, Flandrin G: Ontology-based lymphocyte population description using mathematical morphology on colour blood images. Cell Mol Biol 2006, 52(6):2-15.

9. Cortes C, Vapnik V: Support-Vector Networks. Machine Learning 1995, 20:273-297.

doi:10.1186/1746-1596-8-S1-S32

Cite this article as: Happillon et al:: Label free technologies: Raman micro-spectroscopy and multi-spectral imaging for lymphocyte classification. Diagnostic Pathology 2013 8(Suppl 1):S32. 